\title{
Analysis of Antibody by Real-Valued Special Functions
}

\section{Zhao $\mathrm{B}^{1^{*}}$ and Cao J ${ }^{2}$}

${ }^{1}$ Department of mathematics and Finance, Hanjiang Normal University, Shiyan, Hubei, China

${ }^{2}$ School of Information and Mathematics, Yangtze University, Jingzhou, Hubei, China

*Corresponding author: Zhao B, Department of mathematics and Finance, Hanjiang Normal University, Shiyan, Hubei, China, Fax: +8613028517572, Tel: +8613028517572, E-mail: zhaobin835@nwsuaf.edu.cn

Citation: Zhao B, Cao J (2018) Analysis of antibody by Real-Valued Special Functions. J Antibio Res 2(1): 102

Received Date: September 20, 2017 Accepted Date: April 6, 2018 Published Date: April 8, 2018

\begin{abstract}
Background: Along with the rapid development of genetic engineering technology and antibody engineering technology, humanized monoclonal antibody has been rapidly developed and gradually replaces the rat sourced monoclonal antibody. In this paper, we establish two new logarithmically completely monotonic functions involving the real-valued special functions according to two preferred interaction geometries, necessary and sufficient conditions are presented for one of them to be logarithmically completely monotonic. As a consequence, a sharp inequality involving the real-valued special functions is deduced to solve the problems of genetically engineered antibody.
\end{abstract}

Keywords: Real-valued Special Functions; Genetically Engineered Antibody; Logarithmically Completely Monotonic; Inequality; Psi function

\section{Introduction}

Antibodies have been proven to be indispensable tools for biomedical applications. Different engineered antibodies have been developed for various purposes according to the amino acid sequence and/or spatial structure of protein (Figure 1). At present, it is still difficult to predict the optimal structure of antibodies. Topology knowledge can be important in antibody application as well as transformation. Theoretically, we can obtain desired antibodies by using protein/gene engineering technology. For instance, we can transform the complementarity determining region (CDR) to promote the affinity of the antibody to antigen. Similarly, we could also transform any domain of antibody to make it bind with any desired target. Under this vision, topology is a powerful tool to predict the structure of protein and it will serve to antibody engineering. Our present work tries to explain, and predict, if possible, the change of structure, size and function of antibodies as well as their fragments from a topological perspective.
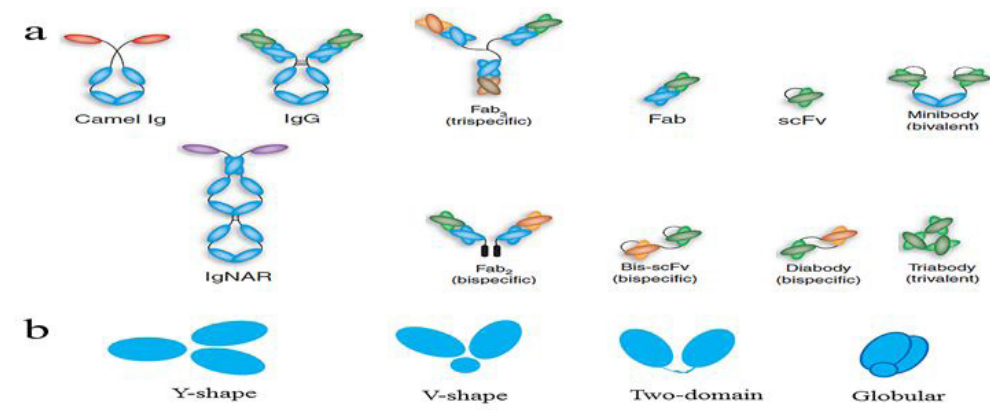

Figure 1: Different antibody formats. a: different antibody or engineered antibodies; b: different shape of antibody

For $\operatorname{Re}(z)>0$ the classical Euler's gamma function $\Gamma$ and psi (digamma) function $\psi$ are defined by

$$
\Gamma(z)=\int_{0}^{\infty} t^{z-1} e^{-t} d t, \psi(z)=\frac{\Gamma^{\prime}(z)}{\Gamma(z)},
$$


respectively. The derivatives $\psi^{(n)}(z)$ for $n \in \mathbb{N}$ are known as polygamma functions. For $\psi(z)[1]$, the following series representations are established :

$$
\begin{aligned}
& \psi(z)=-\gamma-\frac{1}{z}+\sum_{n=1}^{\infty} \frac{z}{n(z+n)}, \\
& \psi(z)=\ln z+\int_{0}^{\infty}\left(\frac{1}{t}-\frac{1}{1-e^{-t}}\right) e^{-z t} d t, \\
& \psi(z)=-\gamma+\int_{0}^{\infty} \frac{e^{-t}-e^{-z t}}{1-e^{-t}} d t
\end{aligned}
$$

where $\gamma=0.577215664901 \cdots$ denotes the Euler's constant.

We next recall that a function $f$ is said to be completely monotonic on an interval $I$, if $f$ has derivatives of all orders on $I$ which alternate successively in sign, that is,

$$
(-1)^{n} f^{(n)}(x) \geq 0
$$

for all $x \in I$ and for all $n \geq 0$. If inequality (1.5) is strict for all $x \in I$ and all $n \geq 0$, then $f$ is said to be strictly completely monotonic [2-5].

The classical Bernstein-Widder theorem [6, p. 160, Theorem 12a] states that a function $f$ is completely monotonic on $(0, \infty)$ if and only if it is a Laplace transform of some nonnegative measure $\mu$, that is,

$$
f(x)=\int_{0}^{\infty} e^{-x t} d \mu(t)
$$

where $\mu(t)$ is non-decreasing and the integral converges for $x>0$.

We recall also that a positive function $f$ is said to be logarithmically completely monotonic on an interval $I$ if $f$ has derivatives of all orders on $I$ and

$$
(-1)^{n}[\ln f(x)]^{(n)} \geq 0
$$

for all $x \in I$ and for all $n \geq 1$. If inequality (1.7) is strict for all $x \in I$ and all $n \geq 1$, then $f$ is said to be strictly logarithmically completely monotonic [7-9].

The antibody structure will be changed when it binds certain targets (Figure 2a), i.e.: antigen, receptor. How to describe the changes in the view of topology? The following cases will explain it in detail.

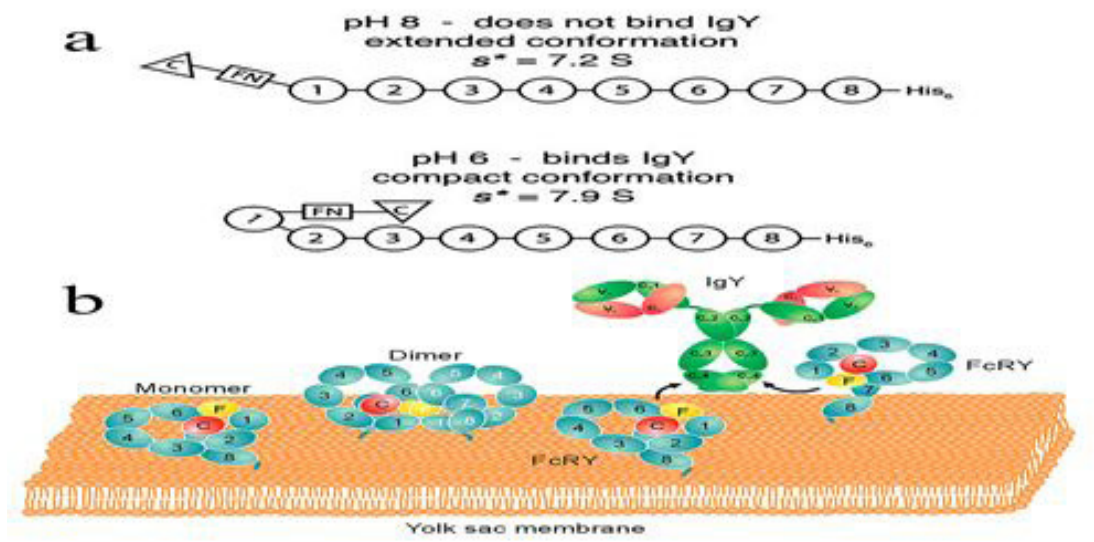

Figure 2: Model of pH-dependent conformational change of FcRY and structures for the FcRY monomer and dimer. a: FcRY has an extended conformation at $\mathrm{pH} 8\left(\mathrm{~s}^{*}=7.2 \mathrm{~S}\right)$ with no predicted interaction between the CysRFNII domains and the CTLDs. At pH 6 the CysR-FNII region folds back and binds to the CTLDs, resulting in a more compact conformation $\left(s^{*}=7.9 \mathrm{~S}\right)$ that is able to bind IgY. b: Likely orientations of FcRY and FcRY-IgY on a membrane. The two FcRY monomers on the Right are shown in an orientation that would allow formation of a 2:1 FcRY-IgY complex. 
It was proved explicitly in and other articles that a logarithmically completely monotonic function must be completely monotonic [8]. In [10], G. D. Anderson et al. proved that the function

$$
g(x)=x(\ln x-\psi(x))
$$

is strictly decreasing and strictly convex on $(0, \infty)$, with two limits

$$
\lim _{x \rightarrow 0} g(x)=1, \lim _{x \rightarrow \infty} g(x)=\frac{1}{2} .
$$

From (1.9) and the monotonicity of $g(x)$, then the double inequalities

$$
\frac{1}{2 x}<\ln x-\psi(x)<\frac{1}{x}
$$

holds for all $x>0$.

In [11, Theorem 1], by using the well-known Binet's formula, H. Alzer generalized the monotonicity and convexity of $g(x)$, that is, the function

$$
g_{\alpha}(x)=x^{\alpha}(\ln x-\psi(x))
$$

is strictly completely monotonic on $(0, \infty)$ if and only if $\alpha \leq 1$.

In [12], D. Kershaw and A. Laforgia proved that the function $[\Gamma(1+1 / x)]^{x}$ is decreasing on $(0, \infty)$ and $x[\Gamma(1+1 / x)]^{x}$ is increasing on $(0, \infty)$. These are equivalent to the function $[\Gamma(1+x)]^{1 / x}$ being increasing and $[\Gamma(1+x)]^{1 / x} / x$ being decreasing on $(0, \infty)$, respectively. In [13,Theorem 5], F. Qi and Ch.-p. Chen generalized these functions. They obtained the fact that for all $x>0$ the function $x^{r}[\Gamma(1+x)]^{1 / x}$ is strictly increasing for $r \geq 0$ and strictly decreasing for $r \leq-1$, respectively.

After the papain digestion, the remained antibody functional part (usually the Fab domain), will be smaller and the structure is also changed (Fig. 1b). These changes can be revealed vividly using topology. Recently [14,Theorem 1], F. Qi, C.-F Wei and B.-N Guo established another excellent result, which states that for given $y \in(-1, \infty)$ and $\alpha \in(-\infty, \infty)$, let

$$
g_{\alpha, y}(x)= \begin{cases}\frac{1}{(x+y+1)^{\alpha}}\left[\frac{\Gamma(x+y+1)}{\Gamma(y+1)}\right]^{1 / x}, & x \in(-y-1, \infty) \backslash\{0\} ; \\ \frac{1}{(y+1)^{\alpha}} \exp [\psi(y+1)], & x=0 .\end{cases}
$$

The function (1.12) is logarithmically completely monotonic with respect to $x \in(-y-1, \infty)$ if and only if $\alpha \geq \max \{1,1 /(y+1)\}$ ; and if $\alpha \leq \min \{1,1 / 2(y+1)\}$, the reciprocal of the function (1.12) is logarithmically completely monotonic with respect to $x \in(-y-1, \infty)$

Antibodies occur spontaneously gathering and forming dimer, polymer, which will influence their functions (Figure 2b). In antibody engineering practice, it urgently needs some measures to overcome this difficulty. From topology perspective, we could understand this issue as follow.

Stimulated by the above results, we put forward the function as follows: for given $y \in(0, \infty)$ and real number $\alpha$, let the function $f_{\alpha, y}(x)$ be defined by

$$
f_{\alpha, y}(x)=\frac{1}{x^{\alpha}}\left[\frac{\Gamma(x+y)}{\Gamma(y)}\right]^{1 / x}, x \in(-y, \infty) \backslash\{0\}
$$

Our first result is contained in the following theorem. 
Theorem 1: For the function (1.13), then the following statements are true:

(1) for any given $y \geq 1$, the function (1.13) is strictly logarithmically completely monotonic with respect to $x \in(-y, \infty) \backslash\{0\}$ if and only if $\alpha \geq 1$;

(2) for any given $0<y<1$, if $\alpha \geq e^{-(1-y)} / y$, then the function (1.13) is strictly logarithmically completely monotonic with respect to $x \in(-y, \infty) \backslash\{0\}$;

(3) for any given $y>0$, the reciprocal of the function (1.13) is strictly logarithmically completely monotonic with respect to $x \in(-y, \infty) \backslash\{0\}$ if and only if $\alpha \leq 0$.

Our second result is presented in the following theorem.

Theorem 2: For any given $y \in[1, \infty)$, let the function $h_{y}(x)$ be defined on $(0, \infty)$ by

$$
h_{y}(x)=\frac{(x+y)^{x}}{\Gamma(x+y) x^{x^{2}}} e^{\left(\frac{1}{2}-\gamma\right) x^{2}-2 x} \exp \left(\sum_{n=1}^{\infty}\left(\frac{x^{2}}{n}+\ln \left(1+\frac{x}{n}\right)^{2 n}-2 x\right)\right),
$$

where $\gamma$ denotes the Euler's constant, then the function (1.14) is strictly logarithmically completely monotonic with respect to $x$ on $(0, \infty)^{\cdot}$

The following corollary can be derived from Theorems 2 immediately.

Corollary 1: For any given $y \geq 1$, the inequality

$$
\frac{(x+y)^{x}}{\Gamma(x+y) x^{x^{2}}} e^{\left(\frac{1}{2}-\gamma\right) x^{2}-2 x}<\frac{1}{\Gamma(y)} \exp \left(-\sum_{n=1}^{\infty}\left(\frac{x^{2}}{n}+\ln \left(1+\frac{x}{n}\right)^{2 n}-2 x\right)\right)
$$

holds for all $x>0$.

\section{Lemma}

In order to prove our main results, we need the following lemmas.

It is well known that Bernoulli polynomials $B_{k}(x)$ and Euler polynomials $E_{k}(x)$ are defined by

$$
\begin{aligned}
& \frac{t e^{t x}}{e^{t}-1}=\sum_{n=0}^{\infty} \frac{B_{n}(x)}{n !} t^{n},|t|<2 \pi \\
& \frac{2 e^{t x}}{e^{t}+1}=\sum_{n=0}^{\infty} \frac{E_{n}(x)}{n !} t^{n},|t|<\pi
\end{aligned}
$$

Respectively [15]. The Bernoulli numbers $B_{n}$ are denoted by $B_{n}=B_{n}(0)$, while the Euler numbers $E_{n}$ are defined by $E_{n}=2^{n} E_{n}(1 / 2)$.

In [16], the following summation formula is given:

$$
\sum_{n=0}^{\infty} \frac{(-1)^{n}}{(2 n+1)^{2 k+1}}=\frac{\pi^{2 k+1} E_{k}}{2^{2 k+1}(2 k) !}
$$

for any nonnegative integer $k$, which implies

$$
B_{2 n}=(-1)^{n-1} \frac{2(2 n) !}{(2 \pi)^{2 n}} \sum_{m=1}^{\infty} \frac{1}{m^{2 n}}, n \in \mathbb{N}
$$

In particular, it is known that for all $n \in \mathbb{N}$ we have

$$
B_{2 n+1}=0, B_{2 n}=(-1)^{n+1}\left|B_{2 n}\right|
$$




$$
E_{2 n+1}=0, E_{2 n}=(-1)^{n}\left|E_{2 n}\right|
$$

And the first few nonzero values are

$$
\begin{aligned}
& B_{0}=1, B_{1}=-\frac{1}{2}, B_{2}=\frac{1}{6}, B_{4}=-\frac{1}{30}, \\
& E_{0}=1, E_{2}=-1, E_{4}=5
\end{aligned}
$$

The Bernoulli and Euler numbers and polynomials are generalized ([18-21]).

(see [17, p.804, Chapter23]).

Lemma 1: For real number $x>0$ and natural number $m[22,23]$, then

$$
\begin{aligned}
& \ln \Gamma(x)=\ln \sqrt{2 \pi}+\left(x-\frac{1}{2}\right) \ln x-x+\sum_{n=1}^{m} \frac{B_{2 n}}{2(2 n-1) n} \cdot \frac{1}{x^{2 n-1}} \\
& +\theta_{1} \frac{B_{2(m+1)}}{(2 m+1)(2 m+2)} \cdot \frac{1}{x^{2 m+1}}, 0<\theta_{1}<1 ; \\
& \psi(x)=\ln x-\frac{1}{2 x}-\sum_{n=1}^{m} \frac{B_{2 n}}{2 n} \cdot \frac{1}{x^{2 n}}-\theta_{2} \cdot \frac{B_{2(m+1)}}{2 m+2} \cdot \frac{1}{x^{2 m+2}}, 0<\theta_{2}<1 ; \\
& \psi^{\prime}(x)=\frac{1}{x}+\frac{1}{2 x^{2}}+\sum_{n=1}^{m} \frac{B_{2 n}}{x^{2 n+1}}+\theta_{3} \cdot \frac{B_{2(m+1)}}{x^{2 m+3}}, 0<\theta_{3}<1 ; \\
& \psi^{\prime \prime}(x)=-\frac{1}{x^{2}}-\frac{1}{x^{3}}-\sum_{n=1}^{m} \frac{B_{2 n}}{x^{2 n+2}}-(2 m+3) \theta_{4} \cdot \frac{B_{2(m+1)}}{x^{2 m+4}}, 0<\theta_{4}<1 .
\end{aligned}
$$

Remark 1: $\theta_{1}, \theta_{2}, \theta_{3}, \theta_{4}$ only depend on natural number $m$.

Lemma 2: For real number $x>0$ and natural number ([24, p. 107, Lemma 3]), we have

$$
\frac{(n-1) !}{x^{n}}+\frac{n !}{2 x^{n+1}}<(-1)^{n+1} \psi^{(n)}(x)<\frac{(n-1) !}{x^{n}}+\frac{n !}{x^{n+1}},
$$

Lemma 3: (see $[1,17])$ For real number $x>0$ and natural number, we have

$$
\begin{gathered}
\ln x=\int_{0}^{\infty} \frac{e^{-t}-e^{-x t}}{t} d t, \\
\frac{\Gamma(n)}{x^{n}}=\int_{0}^{\infty} t^{n-1} e^{-x t} d t \\
\psi^{(n)}(x)=(-1)^{n+1} \int_{0}^{\infty} \frac{t^{n} e^{-x t}}{1-e^{-t}} d t .
\end{gathered}
$$

Lemma 4: Let the sequence of functions for $u(x)$, for $n \in \mathbb{N}$ be defined on $[0, \infty)$ by

$$
u_{n}(x)=\frac{x^{2}}{n}+\ln \left(1+\frac{x}{n}\right)^{2 n}-2 x
$$


the series $\sum_{n=1}^{\infty} u_{n}(x)$ is differentiable on $[0, \infty)$, that is,

$$
\left(\sum_{n=1}^{\infty} u_{n}(x)\right)^{\prime}=\sum_{n=1}^{\infty}\left(\frac{2 x}{n}-\frac{2 x}{n+x}\right) .
$$

Proof: It is obvious that $\sum_{n=1}^{\infty} u_{n}(0)=0$, therefore $\sum_{n=1}^{\infty} u_{n}(x)$ converges at $x=0$. In order to prove (2.16), we need only to show that the inner closed uniform convergence of the series $\sum_{n=1}^{\infty} u_{n}^{\prime}(x)$ on $[0, \infty)$. From (2.15), we have

$$
\sum_{n=1}^{\infty} u_{n}^{\prime}(x)=\sum_{n=1}^{\infty}\left(\frac{2 x}{n}-\frac{2 x}{n+x}\right) .
$$

For any interval $[a, b] \subset[0, \infty)$, we have

$$
\left|\sum_{n=1}^{\infty}\left(\frac{2 x}{n}-\frac{2 x}{n+x}\right)\right| \leq \sum_{n=1}^{\infty} \frac{2 b^{2}}{n^{2}}
$$

for all $x \in[a, b]$. It is easy to check that the series $\sum_{n=1}^{\infty} \frac{2 b^{2}}{n^{2}}$ converges, which and

Weierstrass M-test implies that the series $\sum_{n=1}^{\infty} u_{n}^{\prime}(x)$ is inner closed uniformly convergent on $[0, \infty)$.

Hence the series $\sum_{n=1}^{\infty} u_{n}(x)$ is differentiable on $[0, \infty)$ and the identity (2.17) holds for $x \geq 0$.

The lemma is proved.

Lemma 5: For $0<a \leq 1$ and real number $b$, let the function $Q_{a, b}(x)$ be defined by

$$
Q_{a, b}(x)=\frac{(a x+b)^{x}}{\Gamma(a x+b)}, x \in\left(-\frac{b}{a}, \infty\right) .
$$

If $b \geq a$, then the function (2.19) satisfies

$$
(-1)^{n}\left[\ln Q_{a, b}(x)\right]^{(n)}>0
$$

for all $x \in\left(-\frac{b}{a}, \infty\right)$ and $n=2,3,---$.

Proof: Taking the logarithm of $Q_{a, b}(x)$ yields

$$
\ln Q_{a, b}(x)=x \ln (a x+b)-\ln \Gamma(a x+b),
$$

and differentiating $\ln Q_{a, b}(x)$, then

$$
\left(\ln Q_{a, b}(x)\right)^{\prime}=\ln (a x+b)-a \psi(a x+b)-\frac{b}{a x+b}+1 .
$$


For given integer $n \geq 2$, we get

$$
\left(\ln Q_{a, b}(x)\right)^{(n)}=\frac{(-1)^{n}(n-2) ! a^{n-1}}{(a x+b)^{n-1}}-a^{n} \psi^{(n-1)}(a x+b)-\frac{(-1)^{n-1}(n-1) ! b a^{n-1}}{(a x+b)^{n}}
$$

and, by the identities (2.13) and (2.14), (2.23) can be written as

$$
\left(\ln Q_{a, b}(x)\right)^{(n)}=(-1)^{n} a^{n-1} \int_{0}^{\infty} \frac{e^{-(a x+b) t} t^{n-2}}{1-e^{-t}}\left((1+b t)\left(1-e^{-t}\right)-a t\right) d t
$$

Let $p(t)=(1+b t)\left(1-e^{-t}\right)-a t$ and $q(t)=(1+t)\left(1-e^{-t}\right)-t$. It is easy to check that

$$
q^{\prime}(t)=t e^{-t}>0, t \in(0, \infty)
$$

therefore $q(t)$ is strictly increasing on $(0, \infty)$, and then $q(t)>q(0)=0$.

The following two cases will complete the proof of Lemma 5 .

Case 1: If $0<a \leq 1 \leq b$, then since $q(t)>0$ for $t>0$, we have

$$
a t \leq t<(1+t)\left(1-e^{-t}\right) \leq(1+b t)\left(1-e^{-t}\right) .
$$

which implies $a t<(1+b t)\left(1-e^{-t}\right)$, and then $p(\mathrm{t})>0$ for all $t>0$.

Case 2: If $0<a \leq b \leq 1$, then we get

$$
p^{\prime}(t)=b-a+e^{-t}(b t+1-b) \geq b t e^{-t}>0, t \in(0, \infty),
$$

therefore $p(t)$ is strictly increasing on $(0, \infty)$, and then $p(t)>p(0)=0$.

From (2.24), we know that the inequality (2.20) holds for $x \in(-b / a, \infty)$ and integer .

The lemma is proved.

\section{Proof of Theorems}

Proof of Theorem 1: For $x \neq 0$ and natural number $n$, taking the logarithmically differential into consideration yields

$$
\begin{aligned}
& {\left[f_{\alpha, y}(x)\right]^{(n)}=\frac{1}{x^{n+1}}\left[\sum_{k=0}^{n}(-1)^{k} \frac{n ! x^{n-k} \psi^{(n-k-1)}(x+y)}{(n-k) !}\right.} \\
& \left.-(-1)^{n} n ! \ln \Gamma(y)-\alpha(-1)^{n-1}(n-1) ! x\right]
\end{aligned}
$$

where $\psi^{(-1)}(x+y)$ and $\psi^{(0)}(x+y)$ stand for $\ln \Gamma(x+y)$ and $\psi(x+y)$ respectively.

Furthermore, differentiating $x^{n+1}\left[f_{\alpha, y}(x)\right]^{(n)}$ directly gives

$$
\left\{x^{n+1}\left[f_{\alpha, y}(x)\right]^{(n)}\right\}^{\prime}=(-1)^{n-1} x^{n}\left[(-1)^{n+1} \psi^{(n)}(x+y)-\frac{\alpha(n-1) !}{x^{n}}\right] .
$$

Making use of (2.11) and (2.13) shows that for all $n \in \mathbb{N}$ and any fixed $y>0$, the double inequality 


$$
\begin{gathered}
\int_{0}^{\infty} t^{n-1} e^{-x t}\left[e^{-y t}\left(1+\frac{1}{2} t\right)-\alpha\right] d t=\frac{(n-1) !}{(x+y)^{n}}+\frac{1}{2} \frac{n !}{(x+y)^{n+1}}-\frac{\alpha(n-1) !}{x^{n}} \\
\leq \frac{(-1)^{n-1}}{x^{n}}\left\{x^{n+1}\left[f_{\alpha, y}(x)\right]^{(n)}\right\} \leq \\
\frac{(n-1) !}{(x+y)^{n}}+\frac{n !}{(x+y)^{n+1}}-\frac{\alpha(n-1) !}{x^{n}}=\int_{0}^{\infty} t^{n-1} e^{-x t}\left[e^{-y t}(1+t)-\alpha\right] d t
\end{gathered}
$$

holds for all $x \in(-y, \infty) \backslash\{0\}$ and $\alpha \in(-\infty, \infty)$.

For any fixed $y \in(0, \infty)$, let $u(t)$ and $v(t)$ be defined on $(-\infty, \infty)$ by

$$
u(t)=e^{-y t}\left(1+\frac{1}{2} t\right) \text { and } v(t)=e^{-y t}(1+t)
$$

respectively.

Differentiating $u(t)$ and $v(t)$ directly, we obtain

$$
\begin{aligned}
& u^{\prime}(t)=e^{-y t}\left(\frac{1}{2}-y-\frac{1}{2} y t\right) \\
& v^{\prime}(t)=e^{-y t}(1-y-y t)
\end{aligned}
$$

Therefore, for given $y \in(0, \infty)$ we have

$$
u^{\prime}(t) \begin{cases}>0, & t<1 / y-2 \\ <0, & t>1 / y-2\end{cases}
$$

and

$$
v^{\prime}(t) \begin{cases}>0, & t<1 / y-1 \\ <0, & t>1 / y-1\end{cases}
$$

From (3.6) and (3.7), we conclude that for all $t>0$ we obtain

$$
u(t)>0
$$

and

$$
v(t)\left\{\begin{array}{l}
<1, \quad y \geq 1 \\
\leq e^{-(1-y)} / y, \quad 0<y<1
\end{array}\right.
$$

From (3.3) and (3.8)-(3.9), it is easy to see that

$$
\frac{(-1)^{n-1}}{x^{n}}\left\{x^{n+1}\left[f_{\alpha, y}(x)\right]^{(n)}\right\}\left\{\begin{array}{lll}
>0, & \text { if } \quad \alpha \leq 0 \text { for fixed } y>0 \\
<0, & \text { if } \alpha \geq 1 \text { for fixed } y \geq 1 \\
<0, & \text { if } \alpha \geq e^{-(1-y)} / y \text { for fixed } 0<y<1
\end{array}\right.
$$

for all $n \in \mathbb{N}$ and all $x \in(-y, \infty) \backslash\{0\}$. 
On the one hand, if $x \in(0, \infty)$, then the inequalities (3.10) can be equivalently changed into

$$
\left\{x^{2 k+1}\left[f_{\alpha, y}(x)\right]^{(2 k)}\right\}^{\prime} \begin{cases}<0, & \text { if } \alpha \leq 0 \text { for fixed } y>0 \\ >0, & \text { if } \alpha \geq 1 \text { for fixed } y \geq 1 \\ >0, & \text { if } \alpha \geq e^{-(1-y)} / y \text { for fixed } 0<y<1\end{cases}
$$

and

$$
\left\{x^{2 k}\left[f_{\alpha, y}(x)\right]^{(2 k-1)}\right\}^{\prime} \begin{cases}>0, & \text { if } \alpha \leq 0 \text { for fixed } y>0 \\ <0, & \text { if } \alpha \geq 1 \text { for fixed } y \geq 1 \\ <0, & \text { if } \alpha \geq e^{-(1-y)} / y \text { for fixed } 0<y<1\end{cases}
$$

for $k \in \mathbb{N}$.

From (3.1), then simple computation shows that

$$
\lim _{x \rightarrow 0} x^{n+1}\left[f_{\alpha, y}(x)\right]^{(n)}=0
$$

for all $n \in \mathbb{N}$ and any given $y \in(0, \infty)$. As a result,

$$
x^{2 k+1}\left[f_{\alpha, y}(x)\right]^{(2 k)} \begin{cases}<0, & \text { if } \alpha \leq 0 \text { for fixed } y>0 \\ >0, & \text { if } \alpha \geq 1 \text { for fixed } y \geq 1 \\ >0, & \text { if } \alpha \geq e^{-(1-y)} / y \text { for fixed } 0<y<1\end{cases}
$$

and

$$
x^{2 k}\left[f_{\alpha, y}(x)\right]^{(2 k-1)} \begin{cases}>0, & \text { if } \alpha \leq 0 \text { for fixed } y>0 \\ <0, & \text { if } \alpha \geq 1 \text { for fixed } y \geq 1 \\ <0, & \text { if } \alpha \geq e^{-(1-y)} / y \text { for fixed } 0<y<1\end{cases}
$$

for all $k \in \mathbb{N}$ and all $x>0$.

Therefore, (3.14) and (3.15) imply

$$
(-1)^{n}\left[f_{\alpha, y}(x)\right]^{(n)} \begin{cases}<0, & \text { if } \alpha \leq 0 \text { for fixed } y>0 \\ >0, & \text { if } \alpha \geq 1 \text { for fixed } y \geq 1 \\ >0, & \text { if } \alpha \geq e^{-(1-y)} / y \text { for fixed } 0<y<1\end{cases}
$$

for all $n \in \mathbb{N}$ and all $x>0$.

Hence, if either $\alpha \geq e^{-(1-y)} / y$ for given $0<y<1$ or for given $y \geq 1$, the function (1.13) is strictly logarithmically completely monotonic with respect to $x$ on

$(0, \infty)$, and if $\alpha \leq 0$ for given $y>0$, so is the reciprocal of the function (1.13).

On the other hand, if $x \in(-y, 0)$ for any given $y>0$, then (3.10) implies

$$
\left\{x^{n+1}\left[f_{\alpha, y}(x)\right]^{(n)}\right\}^{\prime} \begin{cases}<0, & \text { if } \alpha \leq 0 \text { for fixed } y>0 \\ >0, & \text { if } \alpha \geq 1 \text { for fixed } y \geq 1 \\ >0, & \text { if } \alpha \geq e^{-(1-y)} / y \text { for fixed } 0<y<1\end{cases}
$$

for all $n \in \mathbb{N}$. 
In view of (3.13), we can conclude that

$$
x^{n+1}\left[f_{\alpha, y}(x)\right]^{(n)} \begin{cases}>0, & \text { if } \alpha \leq 0 \text { for fixed } y>0 \\ <0, & \text { if } \alpha \geq 1 \text { for fixed } y \geq 1 \\ <0, & \text { if } \alpha \geq e^{-(1-y)} / y \text { for fixed } 0<y<1\end{cases}
$$

for $n \in \mathbb{N}$. It is obvious that (3.18) is equivalent to that (3.14) and (3.15) hold for any given $y>0$ and $x \in(-y, 0)$. Therefore, it is easy to prove similarly that (3.16) is also valid on $x \in(-y, 0)$ for any given $y>0$ and all $n \in \mathbb{N}$.

The amino acid of antibody/protein possesses different preferences. Thus we can conduct site-directed mutation to promote the affinity and/or hydrophilic with the prediction of topology. For example, bovine antibodies have an unusual structure comprising a $\beta$-strand 'stalk' domain and a disulphide-bonded 'knob' domain in CDR3 (Figure 3). Attempts have been made to utilize such amino acid preference for antibody drug development.

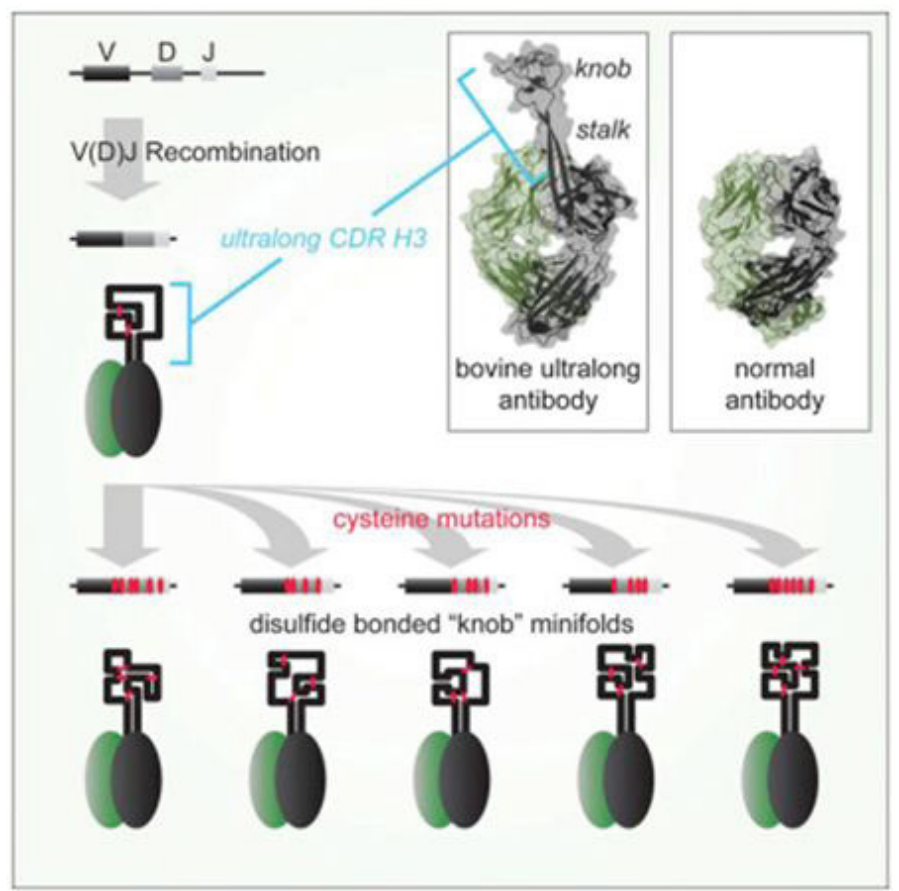

Figure 3: Unique Structural Domain in Bovine IgG antibodies and application

Consequently, the function (1.13) is the same logarithmically completely monotonicity on $(-y, 0)$ as on $(0, \infty)$, that is, if either $\alpha \geq e^{-(1-y)} / y$ for given $0<y<1$ or $\alpha \geq 1$ for given $y \geq 1$, the function (1.13) is strictly logarithmically completely monotonic with respect to $x$ on $(-y, 0)$, and if $\alpha \leq 0$ for given $y>0$, so is the reciprocal of the function (1.13).

Conversely, we assume that the reciprocal of the function (1.13) is strictly logarithmically completely monotonic on $(-y, \infty) \backslash\{0\}$ for any given $y>0$. Then we have for any given $y>0$ and all $x>0$

$$
f_{\alpha, y}^{\prime}(x)=\frac{\ln \Gamma(x+y)-x \psi(x+y)-\ln \Gamma(y)}{x^{2}}+\frac{\alpha}{x}<0,
$$

which implies

$$
\alpha<-\frac{\ln \Gamma(x+y)-x \psi(x+y)-\ln \Gamma(y)}{x} .
$$

By L'H^ospital's rule, we have

$$
\lim _{x \rightarrow 0} \frac{-\ln \Gamma(x+y)+x \psi(x+y)+\ln \Gamma(y)}{x}=0
$$


for any given $y>0$. By virtue of (3.20) and (3.21), we conclude that the necessary condition for the reciprocal of the function (1.13) to be strictly logarithmically completely monotonic is $\alpha \leq 0$.

If the function (1.13) is logarithmically completely monotonic on $(-y, \infty) \backslash\{0\}$

for any given $y>0$, then the inequality (3.19) and (3.20) are reversed for any given $y>0$ and all $x>0$.

By utilizing (2.7) and (2.8), it is easy to see that

$$
\lim _{x \rightarrow \infty} \frac{-\ln \Gamma(x+y)+x \psi(x+y)+\ln \Gamma(y)}{x}=1
$$

for any given $y>0$. In fact, it is not difficult to show that the necessary condition for the function (1.13) to be strictly logarithmically completely monotonic is $\alpha \geq 1$.

The proof of Theorem 1 is completed.

Proof of Theorem 2: Taking the logarithm of $h_{y}(x)$ gives

$$
\ln h_{y}(x)=\ln \frac{(x+y)^{x}}{\Gamma(x+y)}-\ln x^{x^{2}}+\left(\frac{1}{2}-\gamma\right) x^{2}-2 x+\sum_{n=1}^{\infty}\left(\frac{x^{2}}{n}+\ln \left(1+\frac{x}{n}\right)^{2 n}-2 x\right)
$$

Let

$$
\begin{gathered}
\mu(x)=\ln \frac{(x+y)^{x}}{\Gamma(x+y)}, \\
\omega(x)=-\ln x^{x^{2}}+\left(\frac{1}{2}-\gamma\right) x^{2}-2 x+\sum_{n=1}^{\infty}\left(\frac{x^{2}}{n}+\ln \left(1+\frac{x}{n}\right)^{2 n}-2 x\right),
\end{gathered}
$$

then

$$
\ln h_{y}(x)=\mu(x)+\omega(x)
$$

In view of Lemma 4, straightforward calculation gives

$$
\begin{aligned}
& \left(\ln h_{y}(x)\right)^{\prime}=\ln (x+y)+\frac{x}{x+y}-\psi(x+y)-2 t \ln t+2 \gamma x+2-\sum_{n=1}^{\infty}\left(\frac{2 x}{n}-\frac{2 x}{n+x}\right) \\
& =\mu^{\prime}(x)+\omega^{\prime}(x) .
\end{aligned}
$$

By virtue of (1.2), the identity (3.27) is equivalent to

$$
\begin{aligned}
& \left(\ln h_{y}(x)\right)^{\prime}=\ln (x+y)+\frac{x}{x+y}-\psi(x+y)-2 x(\ln x-\psi(x)) \\
& =\mu^{\prime}(x)+\omega^{\prime}(x)
\end{aligned}
$$

By Lemma 5 , we know that $\mu^{\prime}(x)$ is strictly increasing on $(0, \infty)$, which and (1.10) imply the limit of $\mu^{\prime}(x)$ equals 1 as $x \rightarrow \infty$ , therefore

$$
\mu^{\prime}(x)<1
$$

holds for all $x>0$.

We know that $g(x)$ is strictly completely monotonic on $(0, \infty)$, where $g(x)$ is defined by (1.8), hence for given integer $n \geq 0$, the inequality 


$$
(-1)^{n+1}\left(\omega^{\prime}(x)\right)^{(n)}>0
$$

holds for all $x>0$.

And then by using inequality (1.9) and (1.10), we get

$$
-2<\omega^{\prime}(x)<-1
$$

for all $x>0$.

From (3.29) and (3.31), we conclude that

$$
\left(\ln h_{y}(x)\right)^{\prime}=\mu^{\prime}(x)+\omega^{\prime}(x)<0
$$

for all $x>0$. Utilizing Lemma 5 and (3.30), for given integer $n \geq 2$, it is easy to see that

$$
(-1)^{n}\left(\ln h_{y}(x)\right)^{(n)}=(-1)^{n}(\mu(x))^{(n)}+(-1)^{n}(\omega(x))^{(n)}>0
$$

for all $x>0$.

Theorem 2 follows from (3.32) and (3.33).

Thus the proof of Theorem 2 is completed.

\section{Conclusion}

In conclusion, we establish two new logarithmically completely monotonic functions involving the real-valued special functions according to two preferred interaction geometries, and a sharp inequality involving the real-valued special functions is deduced to solve the problems of genetically engineering antibodies. It is necessary to address, many other aspects (such as thermal condition, alkalinity or acidity, adhesion of antibodies) are also playing key roles in antibodies functioning, which could be also understood from bio-mathematical perspective, and such knowledge will be in return useful for biomedical application of antibodies as well as proteins [25-30].

\section{Acknowledgement}

We would like to express my gratitude to all those who helped us during the writing of this article.

\section{References}

1. Erdélyi A, Magnus W, Oberhettinger F, Tricomi FG (1953) Higher Transcendental Functions (Vol I-III) McGraw-Hill, New York.

2. Bernstein S (1929) On absolutely monotonous functions. Acta Math 52: 1-66.

3. Hausdorff F (1921) Summation methods and moment sequences. I. Math J 9: 74-109.

4. Feng Q, Chao-Ping C (2004) A complete monotonicity property of the gamma function. J Math Anal Appl 296: 603-7.

5. Widder DV (1931) Necessary and sufficient conditions for the representation of a function as a La-place integral. Trans Amer Math Soc 33: 851-92.

6. Widder DV (1941) The Laplace Transform, Princeton University Press, Princeton.

7. Atanassov RD, Tsoukrovski UV (1988) Some properties of a class of logarithmically completely monotonic functions, C. R. Acad. Bulgare Sci 41: 21-3.

8. Berg C (2004) Integral representation of some functions related to the gamma function. Mediterr J

Math 1: 433-9.

9. Grinshpan AZ, Ismail MEH (2006) completely monotonic functions involving the gamma and q-gamma functions. Proc Amer Math Soc 134: 1153-60.

10. Anderson GD, Barnard RW, Richards KC, Vamanamurthy MK, Vuorinen M (1995) Inequalties for zero-balanced hypergeometric functions. Trans Amer Math Soc 347: 1713-23.

11. Alzer H (1997) on some inequalities for the gamma and psi functions. Math Comp 66: 373-89.

12. Kershaw D, Laforgia A (1985) Monotonicity results for the gamma function. Atti Accad Sci To- rino Cl Sci Fis Mat Natur 119: 127-33.

13. Qi F, Chen Ch-p (2004) Monotonicity and convexity results for functions involving the gamma function. J Appl Math Sci 1:27-36.

14. Qi F, Wei C-F, Guo B-N (2012) Complete monotonicity of a function involving the ratio of gam- ma functions and applications. Banach J Math Anal 6: 35-44.

15. Guo B-N, Qi F (2010) A property of logarithmically absolutely monotonic functions and the loga- rithmically complete monotonicity of a power-exponential function, Politehn Univ Bucharest Sci Ser A Appl Math. Phys 72: 21-30.

16. Wang Z-X, Guo D-R (2000) Introduction to Special Function. The Series of Advanced Physics of Peking University, Peking University Press, Beijing, China.

17. Abramowitz M, Stegun IA (1970) Handbook of Mathematical Functions with Formulas, Graphs, and Mathematical Tables (9th edn), Applied Mathematics Series, vol.55, National Bureau of Standards, Washington.

18. Guo B-N, Qi F (2002) Generalization of Bernoulli polynomials. Int J Math Educ Sci Technol 33: 428-31.

19. Luo Q-M, Guo B-N, Qi B-N, Debnath L (2003) Generalizations of Bernoulli numbers and polynomials. Int J Math Math Sci 2003: 3769-76. 
20. Luo Q-M, Qi F (2003) Relationships between generalized Bernoulli numbers and polynomials and generalized Euler numbers and polynomials. Adv Stud Contemp Math 7: 11-8.

21. Luo QM, Qi F, Debnath L (2003) Generalizations of Euler numbers and polynomials. Int J Math Math Sci 2003: $3893-901$.

22. Qi F, Guo B-N (2005) Monotonicity and convexity of ratio between gamma functions to different powers, J Indones Math Soc 11: $39-49$.

23. Fichtenholz GM (1964) Differential and integral calculus (Vol II) Berlin, VEB Deutscher Verlagder Wissenschaften.

24. Guo B-N, Qi F (2010) Two new proofs of the complete monotonicity of a function involving the psi function. Bull Korean Math Soc 47: $103-11$.

25. Scott AM, Wolchok JD, Old LJ (2012) Antibody therapy of cancer. Nat rev 12: 278-87.

26. Barbas CF, Languino LR, Smith JW (1993) High-affinity self-reactive human antibodies by design andselection: Targeting the integrin ligand binding site. Proc Natl Acad Sci U S A 90: 10003-7.

27. Miroslava O, Xavier E (2005) Human genetics moves from clinic to bench - and back. Genome Biol, 3: 343.

28. Repp R, van Ojik HH, Valerius T, Groenewegen G, Wieland G, et al. Phase I clinical trial of the bispecific antibody MDX-H210(anti-FcgRI anti-HER-2/neu) in combination with Filgrastim(G-CSF) for treatment of advanced breast cancer. Br J Cancer 89: 2234-43.

29. Alinari L, Lapalombella R, Andritsos L, Baiocchi RA, Lin TS, et al. (2007) Alemtuzumab (Campath-1H) in the treatment of chronic lymphocyticleukemia. Oncogene 26: 3644-53.

30. Burns JC, Mason WH, Hauger SB, Janai H, Bastian JF, et al. (2005) Infliximab treatment for refractory Kawasaki syndrome. J Pediatr 146: 662-7.

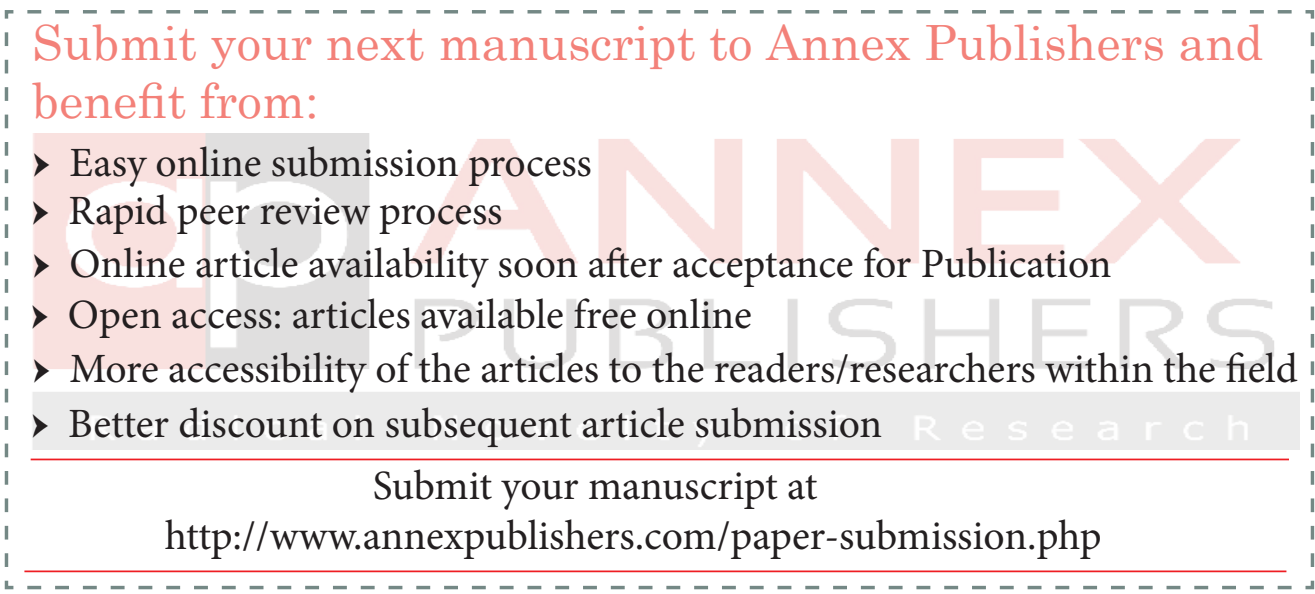

